\title{
Abogados y auxiliares de justicia en el anteproyecto de L.O. del Poder Judicial
}

\author{
por el Dr. ERNESTO PERLA VEIAOCHAGA, \\ Catedrático Titular de Derecho Civil y Derecho Procesal Civil \\ de la Universidad Católica.
}

Antes de entrar al examen de los textos legales, merece destacarse Ia característica común a todos estos puntos para apreciar debidamente el valor de toda reforma sobre la materia. Constituyen entre los elementos del proceso tanto los abogados como los cuxiliares de justicia, elementos personales del proceso. La persona con todas sus virtudes $\mathrm{Y}$ con todos sus defectos, con su inevitable carga humana, interviene en el proceso, e influye decisivamente en él. Por encima de toda ley, y a pesar de todos los esfuerzos de los legisladores, estos elementos personales con su influen. cia unas veces crientarán el proceso hacia su verdadera finalidad contra las deficiencias o el desvío de la ley positiva; otras, desgraciadamente, para hacer inútiles $y$ estériles todos los desvelos de los juristas y los pra pósitos de los legisladores y frustrar el objetivo de que el proceso sea un instrumento de la justicia.

Diversas personas intervienen en el ejercicio o funcionamiento de la potestad jurisáiccional que constituye el proceso. Como éste se produce al solicitarse por la demanda la intervención del poder jurisdiccional para que conozca, tramita y resuelva un conflicto, en él, intervienen ante todo aquellas personas que intentan ante los Tribunales, ya sea como acción - excepción, la tutela jurídica que el Derecho positivo les otorga: éstas son "las partes". Intervienen también las personas físicas de que se tiene que valer el poder jurisdiccional para cumplir su función: éstos son los jueces y los auxiliares de justicia. Ádemás de estas dos clases de personas, intervienen por supuesto otras personas que no son ni interesados ni jueces en el conflicto, sino que sirven para mejorar en alguna forma la tutela jurídica o para hacerla efectiva: son los auxiliares coercitivos, tal la autoridad política, los peritos y estrictamente deben serlo, los testigos que contribuyen con su conocimiento y relato imparcial de los hechos a la comprobación de los mismos. Además, no propiamente como auxiliares, sino como colaboradores de la administración de justicia, intervienen los abo. gados que más bien sirven para poner en relación adecuada y legal a la parte con el juez. Varios elementos todos de orden personal. Todos influ- 
yendo en el proceso, con su moral y su sentido de la vida. Las partes, el juez, los abogados, los auxiliares y los terceros haciendo que el esfuerzo del legislador por una justicia efectiva y rápida, sea posible o imposible, y contra la actitud que ellos adopten toda la ley queda convertida invariablemente en una fría fórmula. Hay que tratar de estas materias haciendo, por lo menos, la reserva de que sobre la reforma del texto de la ley está la reforma de las cosiumbres, que no basta la reforma de la ley escrita, hay que propugnar un mejoramiento moral de los hombres, un mayor sentido de responsabilidad, para que las leyes no sean simples deciaraciones románticas. A la luz de esta premisa, la importancia de la refor$m a$, puede ser relativa, la actual L. O. puede ser tan buena como la que se propone, en lo que respecta, por lo menos, a la materia que nos ocupa.

Así, pues, cuando se trata de un elemento personal, como en el caso que nos ocupa y en otros uspectos de la L. O. debemos tener presente que una ley sobre esta materia, sirve, es buena o mala, sólo en función del hombre a quien le toca ejercer un cargo o jugar un papel en el proceso. Por supuesto que todo esto no elimina la necesidad de una legislación lo más perfecta posible, aunque no sea sino por la fuerza educativa de la ley.

Los abogados.-Nos llevaría fuera de los límites del presente trabajo abordar este tema siempre novedoso y atrayente, tratando de la misión de la abogacía, que es tema cuya consideración nos estimula para desear ser mejores abogados, esto es, luchar contra los elementos que impiden, dificultan o retardan la administración de justicia. Por esto me limitaré al análisis del texto del Anteproyecto.

El título que en el anteproyecto trata de los "Abogados", no contiene sustancialmente más reformas de importancia, sino la consagración de las conquistas que en los últimos años se han venido obteniendo y consagrando en beneficio de los que estamos dedicados a esta nobilísima profesión.

1.-Así, en primer lugar, a la defensa cautiva que adopta la actual L. O., el Art. 437 del Anteproyecto, más amplio, impone que las minutas lleven firmas de letrado, de conformidad con la ley número 11.363, Art. 9\%.

Esta reforma ha sido de gran trascendencia en la vida contractual. Ya no cabe que los contratos que son actos técnicos, estén librados al capricho, a la arbitrariedad y al desconocimiento más absoluto de las leyes que rigen los mismos actos y contratos. Estos errores han sido siem. pre fuente inagotable de posteriore:s controversias judiciales. Errores que producen la nulidad o pueden ser asideros de causales de rescisión; defectuosas redacciones que pueden dar cabida a maliciasas interpretaciones. Todo esto se evita con la intervención del letrado que redacta y autoriza con su firma el instrumento.

Respecto de esto no hay duda alguna. Pero sí se presta a algún comentario la segunda parte del precitado Art. 437, al decir que no es necesaria tal firma: - "si el notario que las redacta tiene también el título 
de abogado". Esta facultad concedida a los notarios-abogados, significa una excepción al principio general de que los notarios no pueden ejercer la abogacía. Por lo mismo debe considerarse como una facultad de excepción, que los notarios deben ejercer muy cautelosamente, como lo hace actualmente la generalidad de ellos.

2:-Declaración del derecho de defensa que tiene el abogado, de la consideración que se merece en el ejercicio de su profesión y otorgamiento de garantías para ello. (Arts. 438-9).

Creo que este principio es de una gran trascendencia y aplicación entre nosotros y que era necesario incluirlo en la L. O. como un expreso reconocimiento del papel que tiene la abogacía en el mundo civilizado $Y$ en el estado de derecho dentro del cual vivimos felizmente.

El Art. 438 dice:-"el abogado tiene el derecho de defensa o prestar "su asesoramiento ante las autoridades judiciales, políticas y administra"tivas sin limitación alguna y ninguna cutoridad política podrá impedir "este ejercicio, bajo responsabilidad".

Se podría añadir en el texto, el abogado tiene el derecho " $y$ el deber", etc... . Lo que completa $\mathrm{y}$ da más fuerza al precepto.

La segunda parte es importante. Es preciso que toda clase de cruta ridades logren desprenderse del anticuado concepto de que el abogado es quien va a embrollar los asuntos e impedir la sinceridad de las partes y la claridad de la cuestión. El abogado, protagonista de las viejas sátiras, como la del abogado Patelín y tantas otras, no trató de representar en los tablados y escenarios, sino al tipo anecdótico. El abogado, que lo es en el concepto cabal, es elemento necesario e imprescindible para el mejor y debido esclarecimiento de los derechos. Si alguna vez, como sucede, tenemos que reconocerlo, algún abogado no está a la altura de su misión, habrá que excluir a ese abogado, en particular, pero no $\alpha$ todos los abogados en general, sin discriminación y precisamente sólo por ser abogados. Unicamente en los regímenes en los que la fuerza prima sobre el derecho el abogado ha sido eliminado o excluído.

Mucho hemos sufrido de esto y negado a veces la defensa por no exponemos $\alpha$ incomprensiones y desaires o con la amarga experiencia de que nuestra intervención sería inútil. Esperamos que con la elevada conducta de todos los abogados, con el efecto educativo que tienen las leyes Y aun compulsivamente desde que la ley declara la responsabilidad por el acto de impedirlas, gocemos en lo venidero de las facilidades necesarias para que en el ejercicio de nuestra profesión nadie se atreva ni pueda decir, "aquí no se necesitan abogados", poniendo a la vez a la puerta, como en la del Paraíso después del pecado, un ángel con una espada para impedirnos la entrada.

Pero, además, el Anteproyecto, en el Art. 439, impone a los jueces $y$ autoridades tratar a los abogados con las consideraciones que merece la profesión que ejercen $\mathrm{y}$ están obligados $\alpha$ proporcionarles todos los medios de ayuda que sean necesarios para el libre ejercicio de la defensa. Incurrirón en responsabilidad los funcionarios $\mathrm{y}$ cutoridades que en cualquier forma impidan a los abogados realizar las gestiones de actividades impuestas por la defensa del asunto que se les haya confiado. 
$3^{\circ}$-Se precisan, aclaran y modernizan las obligaciones de los abogados en los artículos 440,446, 447, 448 y 454, en esta forma:

a) inc. $1^{\circ}$ del Art. 440, se impone la obligación de respetar las normas de ética profesional del Colegio a que pertenezcan.

La vigencia del Código de Etica adoptada en Lima, en Ásamblea General del 17 de noviembre de 1950, queda así incorporado a la L. O. Se incorpora a Ia ley esta honrosa labor por la cual nosotros mismos voluntaria y tenazmente nos pusimos limitaciones al ejercicio de nuestras actividades profesionales y convertimos en normas obligatorias, compulsivas $y$ jurídicas, a las que sólo tenían hasta entonces la calidad de reglas morales.

b) el inc. $5^{\circ}$ del mismo Art. 440, reproduce el texto del Art. 14 del C. de Etica, que establece la relatividad del secreto profesional indispensable mientras un interés superior al que resguarda no imponga la revelación; principio que ès el que consignan las más modernas teorías y con el que estoy conforme porque no es posible negar que hay una jerarquía de valores dentro de la cual el secreto profesional no puede tener sino el lugar que le corresponde en la escala.

c) los artículos 445 al 447, inclusive, establecen el principio de la obligación de los abogados de ceñirse en el concierto de sus honorarios a las tablas mínimas fijadas por el Colegio de Abogados. Sería demasiado largo referimos al asunto. Creo que este sistema ha constituído una de las grandes conquistas conseguidas para los abogados, no sólo desde el punto de vista económico, que también es importante y necesario desde que el profesional tiene el derecho de vivir de su profesión; sino porque da mayor prestigio a los abogados desde que la clientela sabe que la retribución ha de ser la que corresponde a una labor nobilísima y necesaria, a la que le dedican toda su vida los mejores y más preparados elementos de la colectividad.

Pero se da un paso más: ahora por falta de disposiciones de carácter legal estas tasas no pueden aplicarse obligatoriamente por los jueces. Los artículos 446 y 447 establečen que al fijarse el monto de las costas personales (por error dice procesales el Art. 447), no podrán hacerlo en suma inferior a la que establece la referida Tabla de Honorarios. Este es un principio de justicia, pues si el cliente no puede abonar menos de las tasas señaladas, porque el abogado no puede cobrar menos, es justo que en ningún caso se le reintegre al victorioso una suma menor, porque así se propicia el intentor aventuras judiciales con la seguridad de una verdadera impunidad, desde que la condena al pago de costas, por sus reducidas proporciones no tiene el carácter de una verdadera sanción.

d) el Art. 448, de conformidad con el acuerdo de la Ascrmblea General de los miembros del Colegio, otorga el goce de los beneficios sociales a los abogados que perciben honorarios periódicos de sus clientes.

e) el Art. 454, reprime el ejercicio ilegal de la abogacía, declarando que quien lo practica incurre en delito de ejercicio ilícito de la profesión, según el Ārt. 11 de la ley 11.363.

Así, pues, todos los beneficios que las leyes otorgan a los abogados quedan incorporados $\alpha$ la ley, $y$ el Anteproyecto en esta parte es inobjetable. 
Pasando a tratar de los auxiliares de justicia, el Anteproyecto, al adoptar esta última denominación, lo que no hizo la ley vigente, sigue es verdad la clasificación común de la doctrina, pero es evidente que, como queda dicho, hay otros auxiliares, tales como los peritos, la autoridad politica y hasta los testigos.

También es preciso hacer notar la importancia que tienen estos auxiliares. No debe estimarse por un momento, que son elementos de segundo orden. No. Ellos intervienen decisivamente en el proceso. Por lo menos, esto sucede en la actualidad y en todos los regímenes, especialmente en los que, como en el nuestro, predomina el sistema escrito. Pero no ha sido siempre asi. Mientras el proceso se mantuvo en el período primitivo u originario y se reducia a una función verbal y decisoria por el poder omnímodo del sacerdote-rey-juez, radicado en una misma persona, no fué necescrio el auxiliar. Pero, jcuánto ha variado desde entonces el proceso! Por razón de estas variaciones, los auxiliares han ido adquiriendo decisiva importancia. - En este proceso evolutivo, desde el punto de vista de los auxiliares, siguiendo en general al tratadista español, Dr. L. Prieto Castro, notamos las siguientes etapas:

1) se diferencian las labores de rey-sacerdote y magistrado; quien: las ejerce las delegá en otras personas, y como consecuencia desaparece el carácter absoluto de la decisión, apareciendo la revisión, la apelación - las instancias. Esto hizo necesaria la forma escrita en reemplazo de la primitiva forma simplemente verbal. La forma escrita, exige ya al escribano.

2) por la misma razón se hace preciso custodiar y guardar el material del juicio, lo que nosotros llamaríamos, los autos. Se requiere una persona que tenga esta función o cometido.

3) es preciso que los actos del juez y de las partes sean autenticados para garantía de ambos. Ha comenzado esto con la intervención de testigos que dan fe de los actos, pero posteriormente con esa. influencia. tan beneficiosa que el Derecho de Ia Iglesia tuvo y tiene en todas las órdenes del Derecho, con la Decretal del Papa Inocencio III (1216), se adscribe a los Tribunales un funcionario que es el depositario de la fe pública.

4) por último, con la complicación aparece la tecnicidad del proceso que requiere elementos también preparados para estas funciones técnicas. Se ha objetado esto afirmándose que basta el juez para la función técnica y que no se requiere otro técnico. Más aún, entre nosotros, tratúndose de los actuarios, he tenido ocasión de oír decir y no una sola vez, sino varias que mejor es el que el actuario no sepa mucho, na tenga gran. experiencia porque entonces puede llegar a sustituir al juez en su función. Esto lo considero un grave error de apreciación. El actuario tiene y debe tener conocimientos técnicos, no para sustituir al juez, por supuesto, sino porque, su función es de carácter técnico, debe "dar cuenta" y esto no lo puede hacer debidamente sino quien tiene los conocimientos necesarios $Y$ Iá experiencia que se requiere para conocer la cuestión, pues los conocimientos técnicos le otorgan los medios parł́ exponer debidámente el caso. inclusive el léxico científico, y aun para proponer la solución y para re. dactarla fuera de que en todos los demás actos de su fúnción, diligencias. 
trómites, etc.... no puede ser un empírico, sino conocer lo que debe hacer, cómo y por qué hacerlo.

Insisto en que debe ser un elemento esencialmente técnico, porque esta premisa me lo permite en la apreciación y enjuiciamiento de la ley propuesta, y llegar a determinadas conclusiones.

Es evidente que admitiendo como premisa de toda legislación sobre auxiliares de justicia y particularmente sobre actuarios, que es el caso en que se plantea la mayor discusión y a los que únicamente me referiré, y a la más trascendental reforma, partiendo digo, de la premisa de que se requiere que sean elementos técnicos se suele optar por dos soluciones: Una radical: los actuarios actuales deben ser sustituídos en todas sus funciones por los Secretarios Letrados; quedan abolidos los actuarios. Otra solución ecléctica: los actuarios actuales deben ser sustituídos por los Seeretorios Letrados de Juzgado en las funciones que significan y requieren precisamente conocimientos técnicos, quedando así relegados los actuarios a las simples funciones mecónicas o manuales. Se opta así por separar y distinguir las funciones técnicas de las funciones no técnicas. Las primeras serán propias de Ios Secretarios Letrados, las segundas de los actuarios.

Esta última es la solución del "Anteproyecto". Tiene la ventaja de no deconocer los derechos adquiridos por estos funcionarios que han ganado su título, que ostentan reconocido por el Estado de por vida; $y$, con este reconocimiento, la secuela de que después de muchos años de fatiga, $\alpha$ veces $y$ generalmente en toda una vida dedicada $\alpha$ estas funciones no es posible suprimirlos de un plumazo, como sucede cuando invocado, se derrumba un edificio respetable para facilitar el trónsito $\mathrm{y}$ no obligar $\alpha$ dar una curva a los voraces automotores, se le hecha abajo y construye una pista donde, a su vez, se han de producir accidentes, es verdad que de otra clase, pero siempre accidentes que lamentar, porque el mal no estaba en los peligros de las curvas del trayecto, ni después en la tentadora ruta, sino en el vértigo de la velocidad. Algo de esto podría pasar al abolirse la institución de los actuarios.

El Anteproyecto aplicando la segunda solución en su capítulo III. establece los Secretarios Letrados de Juzgado. Los requisitos para ejercer el cargo y sus obligaciones (Art. 323), son similares a las de los Secretarios de Corte. Pero es de destacar que se les asigna obligaciones que hoy corresponden en parte $\alpha$ los jueces de Primera Instancia y en parte a los escribanos actuarios. De los primeros son las obligaciones que se les im. pone por el Art. 271, aplicable el inc. $4^{\circ}$, Art. 324, esto es, las indicadas en los incisos $2^{\circ}, 11^{\circ}, 12^{\circ}, 13^{\circ}, 15^{\circ}$ y $16^{\circ}$ del Art. 269.

Se pueden agrupar así las funciones que se asignan a los Secretarios Letrados: a funciones jurisdiccionales; deciden o proveen, los escritos sobre los que deben recaer simples decretos (inc. 3ㅇ, Art. 324).

a) A este respecto caben algunas observaciones. En primer lugar, la dificultad que se puede presentar para la calificación de la resolución que corresponde si es cuto o decreto. Fn segundo lugar, debe tenerse en consideración lo instituído por el Art. 272, en cuanto $\alpha$ los recursos legales atinentes a las resoluciones que dicta el Secretario Letrado. Se establece que procede contra ellas el recurso de reposición en lo que no hay modi. 
ficación del sistema del actual C. de P. C., desde que se trata de decretos. Las reposiciones son resueltas directamente por el Juez. Esto es alterar la esencia misma de la reposición, recurso por el cual el mismo juez que ha expedido una resolución conoce el recurso de reclamo y lo decide: según el anteproyecto es una persona distinta la que conoce el recurso, lo que le resta su carácter de reposición, para convertirse virtualmente en una apelación. Sigue el procedimiento; el cuto que resuelve la reposición. es apelable en un sólo efecto, en la forma vigente, esto es con copias. La Corte debe resolver bajo la prohibición absoluta de solicitar los autos, y sólo teniendo la facultad de ordenar que se amplíen las copias presentadas. Esta es una modificación de orden procesal y no de derecho orgánico, $y$, por lo tanto, está fuera del lugar que le corresponde.

b) Funciones administrativas: llevar los libros de certificados de depósitos, la contabilidad conforme al Art. 100 de la ley 7964, de tutelas y curatelas, Registros de correcciones disciplinarias al personal del Juzgado, de circulares y otras comunicaciones, examinar el libro copiador de resoluciones, formular la tabla semanalmente (incisos 11,12 y 13), y suministrar los datos necesarios para la estadística judicial e inspeccionar los oficios de los actuarios (incisos 15 y 16), esto es lo que hoy hace el Vocal Visitador.

c) Funciones de ejecución: acompañar al juez a las diligencias que se realicen fuera del local del Juzgado, cuando el Juez lo disponga (Art. 324,

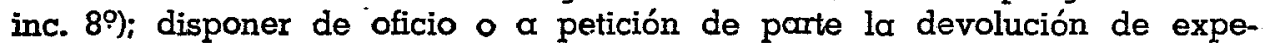
dientes, imponer orden en las diligencias, asistir $y$ tomar parte en todas las actividades que el juez le indique relacionadas con el despacho judicial. De las obligaciones que hoy corresponden a los actuarios se toman las demás obligaciones que fijan en el Art. 324, para los Secretarios Letrados de Juzgado.

Cabe, pues, considerar a la luz de nuestra propia experiencia esta propuesta solución. Equivale en buena cuenta $\alpha$ poner nuevamente, sobre el tapete la cuestión de la conveniencia o desventaja de establecerse entre nosotros el Secretariado Letrado.

Encuentro un primer inconveniente de orden práctico. Tal reforma no podrá funcionar sin un aumento muy apreciable en la escala de los haberes de los magistrados.

Debiendo ser legalmente inferior el sueldo del Secretario Letrado que el del Juez, pocos estarían dispuestos a dedicarse a él, con la escala actual. En fin, si la diferencia es grande entre ambos emolumentos, nadie se dedicará a este puesto, pues resulta muy reducida la remuneración; y si la diferencia es pequeña, mós vale crear más jueces.

En todo caso, sería mejor que crear los Secretarios de Juzgado, establecer con pequeño aumento en el presupuesto, un mayor número de jueces de Primera Instancia, así sería posible que ellos ejercitaran un mayor control sobre los auxiliares de justicia y tuviesen una más directa $\mathrm{y}$ personal intervención en las actuaciones del proceso y mayor control sobre los actuarios; que es uno de los principales objetivos de la reforma.

Se puede objetar que el cargo de Secretario de Juzgado constituye: el primer grado de la escala de la carrera judicial. Pero esto supone tam- 
biến la reforma de las díspasiciones relativas a la provisiớn de los cargos judiciales; otro escollo formidable de la reforma. Con esto más, que el aseenso no es obligatorio, pues conforme al Art. 48, se puede ingresar a la carrera judicial en cualquiera de sus grados. Por último, la inamovilidad de la carrera hace remotas las yacantes juliciales, no siendo por todas estas razones un verdadero incentivo para el cargo de Secretario como primer peldaño de la carrera judicial.

La segunda consideración que me suscita esta reforma es la escasa importancia que tendró la labor del Secretario de Juzgado. Según he expuesto de las varias funciones que le corresponden, las jurisdiccionales se limitan a poner los decretos. No creo que para dictar éstos, sea necesario crear y mantener una judicatura. Para las funciones administrativas de llevar los libros, inspeccionar las oficinas de los actuarios y archivar las comunicaciones no justifica la necesidad de crearlos. Para las inspecciones existe el vocal visitador. En todo caso podrá disponerse que las visitas se hagan con mayor asiduidad y se podría dotar a cada Juzgado de un em. pleado que tuviera las funciones de archivero, para la otra parte, escasísimos documentos y comunicaciones que suele recibir. Respecto a las funciones de ejecución, en cuanto acompañar al Juez en las diligencias me parece totalmente inútil, y considero perjudicial y contraproducente $y$ contra el principio de inmediación que sea el Secretario y no el Juez que va a expedir la sentencia quien actúe las confesiones, comparendos y demás diligencias probatorias.

La tercera objeción es que los Secretarios de Juzgado por su propia jerarquía sólo podrón ser los abogados recientemente egresados de la Facultad $\mathrm{y}$, por lo mismo, carentes de experiencia $\mathrm{y}$ de la autoridad que sería necesaria para, efectivamente, dictar los proveídos o ejercer control sobre el actuario ordinariamente más experimentado y que para explicar - justificar las deficiencias en que puede incurrir en el servicio que presta, sostiene el recargo de sus labores.

La cuarta objeción, es una consecuencia de la anterior: la responsabilidad del juez que hoy queda atemperada por la del actuario, mañana quedaría repartida entre el juez, el secretario y el actuario, quienes se podrían hacer recíprocamente cargos sobre de quién depende el retardo en la administración de justicia y los errores o inadvertencias en que se puede incurrir en el procedimiento.

Pero no debe limitarse nuestro examen a la labor negativa de criticar sin construir. Creemos que se requiere una reforma; que los males de que adolece la administración de justicia constituyen uno de los más gran. des $y$ urgentes problemas nacionales y que la cuestión que nos ocupa, es una de las que conjuntamente con el sistema de provisión de los cargos judiciales $y$ su adecuada retribución, tiene mayor importancia y trascendencia para conseguir el anhelo colectivo de una mejor y más pronta ad. ministración de justicia.

Pero creemos que el Secretariado judicial no es la solución y nos atrevemos a sugerir otros: aumentar el número de jueces de Primera Instancia en proporción a la población, como lo hace el Reglamento de Jueces de Paz. 
Es evidente que, en Lima, siete jueces es un número muy reducido para atender todo el Despacho judicial de las cuestiones civiles en una. población que no puede bajar de un millón de habitantes. Los jueces han. estado recibiendo últimamente no menos de tres mil causas civiles en cada turno. Cada una de ellas requiere la intervención del juez, precisamente en la etapa más larga, la de las pruebas y los comparendos. Y hay que pensar cuóntas de estas diligencias imponen las tres mil causas. No es posible exigir al juez que pueda atender a todas en la medida que es aconsejable y conveniente, pero tompoco se debe dividir su responsabili. dad que debe ser considerada única e indivisible. Por esto es que la solución es la creación de un mayor número de Juzgados de Primera Instancia, en una proporción variable en relación con la población. Estos datos rela. tivos a lima, creo que puedan aplicarse con la necesaria relatividad $\alpha$ cualquier otra circunscripción judicial.

Además, debe propiciarse que los abogados se interesen por desempeñar los cargos de actuarios. Para esto se podría consignar en la L. O. un precepto similar al de la Ley de Notariado, por el cual los abogados tienen preferencia a los que no lo son para llenor las vacantes que se produzcan o al crearse nuevas plazas. Con esta norma se ha conseguido que en el transcurso de algunos años todos los notarios prácticamente sean abogados. Lo mismo podría suceder tratándose de los actuarios, desde que no es una actividad poco fructífera desde el punto de vista económico.

Por último, este sistema se mejoraría estableciendo una mesa de partes en cada Juzgado, en la cual debería presentarse los diversos recursos, haciéndose constar la fecha de su presentación y donde debería lle. varse un libro de "tomas de razón", similar al que existe en las Mesas de Partes de las Cortes. 